\title{
Automatic Selection of Multiple Images in the Frontier Field Clusters
}

\section{Guillaume Mahler, Johan Richard, Benjamin Clément, David Lagattuta and Vera Patricio}

\author{
Centre de Recherche Astrophysique de Lyon, \\ Université Lyon 1, Lyon, France \\ email: guillaume.mahler@univ-lyon1.fr
}

\begin{abstract}
Probing the central mass distribution of massive galaxy clusters is an important step towards mapping the overall distribution of their dark matter content. Thanks to gravitational lensing and the appearance of multiple images, we can constrain the inner region of galaxy clusters with a high precision. The Frontier Fields (FF) provide us with the deepest HST data ever in such clusters. Currently, most multiple-image systems are found by eye, yet in the FF, we expect hundreds to exist. Thus, in order to deal with such huge amounts of data, we need to develop an automated detection method. I present a new tool to perform this task, MISE (Multiple Image SEarcher), a program which identifies multiple images by combining their specific properties. MISE allows us to confirm or reject multiple images identified visually, but also detect new multiple-image candidates in MACS0416 and A2744, giving us additional constraints on the mass distribution in these clusters. A spectroscopic follow-up of these candidates is currently underway with MUSE.
\end{abstract}

Keywords. Frontiers Fields, gravitational lensing, multiple images, lens modelling, MACS0416

\section{Motivation}

Strong lensing by massive clusters produces multiple images of background galaxies which can be used to better constrain the total mass distribution in the cluster cores. Depending on the depth of the observations, the number of detectable multiple images grows, from 36 images/arcmin ${ }^{2}$ in shallow CLASH data (Zitrin et al. 2012) to 90 images/arcmin ${ }^{2}$ in deep HFF data (Jauzac et al. 2014). Future observations, such as those taken by JWST, will be even deeper, so we will need to go further than traditional visual inspection by developing automatic selection methods based on quantitative criteria.

\section{Criteria}

Different criteria can be used to identify multiple-image systems, based on both photometric and morphological aspects of the galaxies. One important criterion, the SED (Spectral Energy Distribution) comparison, uses color information, similar to the criterion presented by Wang et al. (2014). To accurately measure the photometry and colors of candidate multiple images and to optimize the detection of very faint sources, we first pre-process the Hubble images by subtracting the smooth cluster light distribution using a running median window of 1.3". After this subtraction, we then compare the SEDs of all objects, looking for matched pairs which could be multiple images of the same galaxy. To compare objects $\mathrm{A}$ and $\mathrm{B}$ we use a $\chi_{\nu}^{2}$ minimization with a scaling factor $\alpha$ :

$$
\chi_{\nu}^{2}=\frac{1}{N-1} \min _{\alpha}\left(\sum_{i=0}^{N} \frac{\left(f_{i}^{A}-\alpha f_{i}^{B}\right)^{2}}{\sigma_{i}^{A^{2}}+\alpha^{2} \sigma_{i}^{B^{2}}}\right)
$$




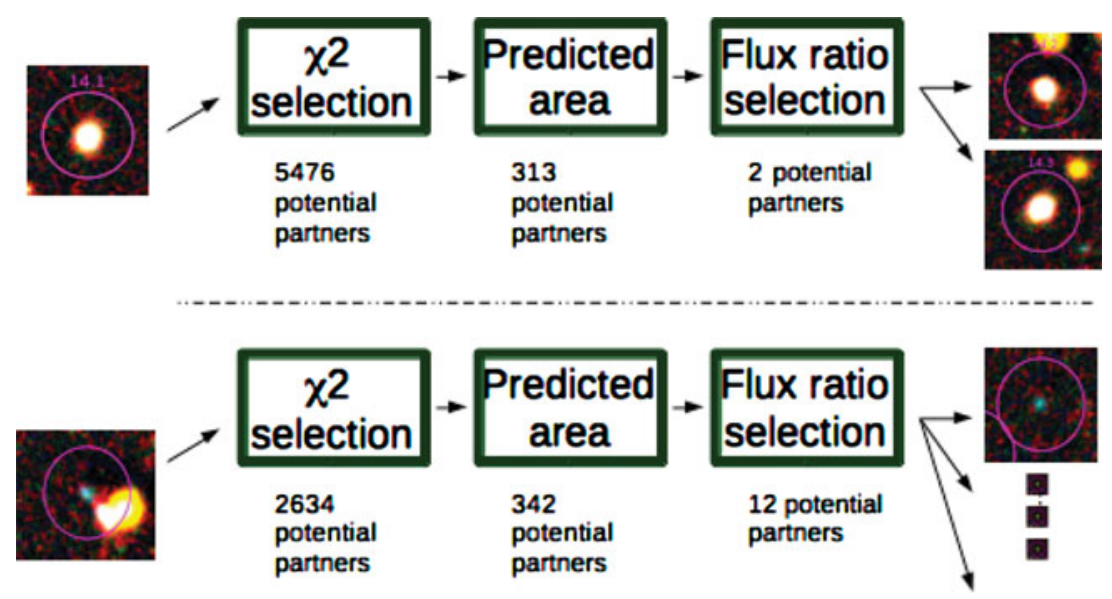

Figure 1. Two examples of the selection process of multiple-image candidates. The top panels show system 14 from Jauzac et al. 2014. The bottom panels show a potential new system. For each selection made, you can see below the number of potential partners remaining.

where $\mathrm{N}$ is the number of photometric filters used in the SED, and $f_{i}$ and $\sigma_{i}$ are the measured flux and error in filter $i$.

To reduce the possible number of matches we only keep pairs with a $\chi_{\nu}^{2}$ value less than 6 and an $\alpha$ factor between 0.1 and 10. These limits are also used by Lam et al. (2014) to check the predicted magnitudes of their model (their figure 27).

\section{Perspectives}

To better improve image matching, we also look at morphological parameters such the Gini coefficient (Lotz et al. 2004) which seems, according to our preliminary tests, to be conserved by lensing. By combining all of these criteria with MUSE spectroscopy, we will be able to confirm real multiple-image systems, including much sought after high-redshift Lyman alpha emitters. However, before using MISE on observed data, we will first test the process on simulated clusters at FF depths (ARES, Meneghetti et al. 2010; HERA, Giocoli et al. 2012) in order to improve the method.

\section{References}

Giocoli, C., Meneghetti, M., Bartelmann, M., Moscardini, L., \& Boldrin, M. 2012, MNRAS, 421, 3343

Lam, D., Broadhurst, T., Diego, J. M., Lim, J., Coe, D., Ford, H. C., \& Zheng, W. 2014, ApJ, 797,98

Jauzac, M., Clément B., Limousin, M., Richard, J., Jullo, E., Ebeling, H., Atek, H., Kneib, J.-P., Knowles, K., Natarajan, P., Eckert, D., Egami, E., Massey, R., \& Rexroth, M. 2014, MNRAS, 443, 2, p.1549-1554

Johnson, T. L., Sharon, K., Bayliss, M. B., Gladders, M. D., Coe, D., \& Ebeling, H. 2014, ApJ, 797,48

Lotz, J. M., Primack, J., \& Madau, P. 2004, ApJ, 128, 163

Meneghetti, M., Rasia, E., Merten, J., Bellagamba, F., Ettori, S., Mazzotta, P., \& Dolag, K. 2010, A\& $A, 514,93$

Wang, X., Hoag, A., Huang, K.-H., Treu, T., Bradač M., Schmidt, K. B., Brammer, G. B., Vulcani, B., Jones, T. A., Ryan, R. E., Jr. Amorín R., Castellano, M., Fontana, A., Merlin, E., \& Trenti, M. 2015, ApJ, 811, 29W 